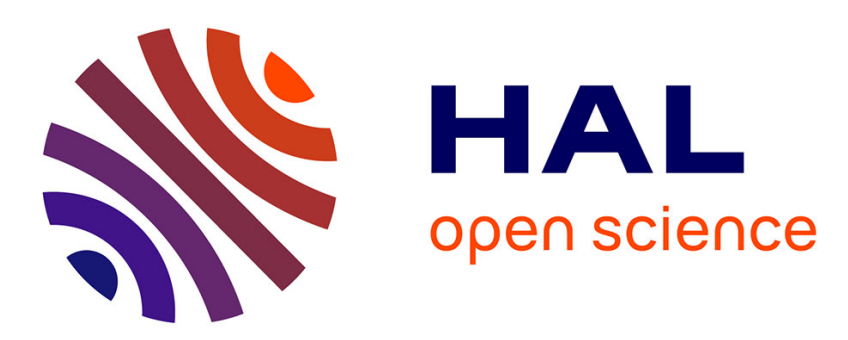

\title{
Coupling of an aeroacoustic model and a parabolic equation code for long range wind turbine noise propagation \\ Benjamin Cotté
}

\section{- To cite this version:}

Benjamin Cotté. Coupling of an aeroacoustic model and a parabolic equation code for long range wind turbine noise propagation. Journal of Sound and Vibration, 2018, 422, pp.343-357. 10.1016/j.jsv.2018.02.026 . hal-01774235

\section{HAL Id: hal-01774235 \\ https://hal-ensta-paris.archives-ouvertes.fr/hal-01774235}

Submitted on 23 Apr 2018

HAL is a multi-disciplinary open access archive for the deposit and dissemination of scientific research documents, whether they are published or not. The documents may come from teaching and research institutions in France or abroad, or from public or private research centers.
L'archive ouverte pluridisciplinaire HAL, est destinée au dépôt et à la diffusion de documents scientifiques de niveau recherche, publiés ou non, émanant des établissements d'enseignement et de recherche français ou étrangers, des laboratoires publics ou privés. 


\title{
Coupling of an aeroacoustic model and a parabolic equation code for long range wind turbine noise propagation
}

\author{
B. Cotté \\ Institute of Mechanical Sciences and Industrial Applications (IMSIA), ENSTA ParisTech, \\ CNRS, CEA, EDF, Université Paris-Saclay \\ 828 bd des Maréchaux, 91120 Palaiseau, France
}

\begin{abstract}
This study proposes to couple a source model based on Amiet's theory and a parabolic equation code in order to model wind turbine noise emission and propagation in an inhomogeneous atmosphere. Two broadband noise generation mechanisms are considered, namely trailing edge noise and turbulent inflow noise. The effects of wind shear and atmospheric turbulence are taken into account using the Monin-Obukhov similarity theory. The coupling approach, based on the backpropagation method to preserve the directivity of the aeroacoustic sources, is validated by comparison with an analytical solution for the propagation over a finite impedance ground in a homogeneous atmosphere. The influence of refraction effects is then analyzed for different directions of propagation. The spectrum modification related to the ground effect and the presence of a shadow zone for upwind receivers are emphasized. The validity of the point source approximation that is often used in wind turbine noise propagation models is finally assessed. This approximation exaggerates the interference dips in the spectra, and is not able to correctly predict the amplitude modulation.
\end{abstract}

Keywords: Wind turbine noise, parabolic equation, atmospheric propagation, aeroacoustic sources, point source approximation

Email address: benjamin.cotte@ensta-paristech.fr (B. Cotté) 


\section{Introduction}

Noise from wind turbines can be perceived at large distances, of the order of one kilometer or more, and is characterized by amplitude modulations that vary depending on the receiver position and atmospheric conditions [1, 2, 3]. In

5 order to predict this noise, it is necessary to accurately model the aeroacoustic sources that are generally dominant for a modern wind turbine [1, 4, 5, as well as the propagation phenomena in the atmospheric boundary layer [6, 7]. This raises the problem of coupling source and propagation models, since wind turbine blades are moving and extended noise sources of complex directivity.

It is common in the literature to use the point source approximation in wind turbine noise propagation models. The noise is supposed to be produced by a monopole of specified power located at the rotor center. The wind turbine is thus represented as an omnidirectional point source that can easily be included in various propagation codes. This approach has been used for instance in the 15 ray-tracing calculations of Ref. [8, and in the parabolic equation calculations of Refs. 9, 10]. Generally, the power spectrum of the source is obtained from sound pressure level measurements at a reference location in accordance with IEC 61400-11.

To justify this approach, many studies refer to the work of Makarewicz [11, who claims that the point source approximation is valid when the distance between the receiver and the wind turbine is at least twice the blade length. This conclusion is drawn from a fairly simple model, that assumes that the wind turbine blades can be reduced to a single point source at the tip of each blade, and that neglects the influence of the ground and of the inhomogeneity 25 of the atmosphere. On the other hand, Heutschi et al. 3] have shown that wind turbines have to be viewed as extended sources to correctly account for the ground effect. This is clearly seen in their noise spectra, that present interference dips that are too strong when the point source approximation is used.

The effect of wind speed gradients in the atmospheric boundary layer is known to have an influence on both the noise sources and their long range 
propagation. van den Berg [12] has shown that situations with a high wind shear at night are commonly encountered. In these situations, the difference of wind speeds between the bottom and top of the rotor can be significant. This means that the blade angles of attack will vary during one rotation, which could lead to partial or complete detachment of the boundary layer, as shown in the measurements of Bertagnolio et al.. [13. This modification of the noise source mechanisms is one of the possible explanations of the strong amplitude modulation of wind turbine noise that can be measured in the far-field [2]. Wind speed gradients also influence acoustic propagation through refraction effects.

40 It is well known that refraction tends to cause a channelling of noise downwind, and creates a shadow zone upwind whose size depends on frequency, as shown for instance by Hubbard and Shepherd [6].

There is currently a strong interest in developing propagation models that consider extended noise sources in order to correctly predict amplitude mod-

45 ulation phenomena that are due to a combination of emission and propagation effects. Several studies propose approaches based on ray-tracing methods [14, 15, 16], while other consider parabolic equation methods [17, 18]. The objective of this study is to couple a source model based on Amiet's theory and a parabolic equation $(\mathrm{PE})$ code in order to model the emission and propagation of wind turbine noise in an inhomogeneous atmosphere and to assess the validity of the point source approximation. Compared to other studies, the originality of the coupling approach consists in using the backpropagation method to preserve the directivity of the aeroacoustic sources. Note that the word coupling is used throughout the article in the computer programming sense, meaning that there is a strong interdependence between the source and propagation codes. It does not mean that there is a feedback from the propagation model on the source radiation.

Both trailing edge noise and turbulent inflow noise are included in the aerocoustic source model. Although a frequency-domain model based on Amiet's theory is considered in this article, based on our previous work 4], the method can be straightforwardly extended to any other frequency-domain source model, 
such as the so-called BPM semi-empirical model that is widely used in wind turbine noise prediction studies [1, 19], or the combined aeroelastic-aeroacoustic model recently proposed by Bertagnolio et al. 5, that includes an improved model for separation and stall noise compared to the original BPM model.

The organization of the paper is as follows. In Section 2 , the source and propagation models are described, as well as the proposed coupling method. Then, the model, hereafter called Amiet-PE model, is validated in Section 3 by comparison with an analytical model for the propagation over a ground of finite impedance. Finally, results are presented in Section 4 to show the influence of ground and meteorological effects in different directions of propagation, and test the validity of the point source approximation.

\section{Description of the coupled model}

\subsection{Aeroacoustic source model based on Amiet's theory}

We recently proposed in Ref. 4 a model of trailing edge noise and turbulent inflow noise for wind turbines based on Amiet's theory [20, 21, 22]. For an airfoil of chord $c$ and span $L$ that is fixed relative to a far-field receiver, and for aspect ratio $L / c$ greater than about 3 , the power spectral densities (PSD) of the acoustic pressure can be written in the general form:

$$
S_{p p}^{F}\left(\mathbf{x}_{\mathbf{R}}, \omega\right)=A\left(\mathbf{x}_{\mathbf{R}}, \omega\right) \Pi\left(\mathbf{x}_{\mathbf{R}}, \omega\right)\left|\mathcal{I}\left(\mathbf{x}_{\mathbf{R}}, \omega\right)\right|^{2}
$$

with $\mathbf{x}_{\mathbf{R}}$ the position of the far-field receiver, $A$ a coefficient that depends on the geometry and the angular frequency $\omega, \Pi$ a statistical function and $\mathcal{I}$ an aeroacoustic transfer function. These functions depend on the noise generation mechanism considered and are detailed in Refs. [22, 4]. The superscript $F$ in Equation (1) refers to the fixed airfoil.

The main input parameter of the model is the $\Pi$ function. For turbulent inflow noise, corresponding to the interaction between the incident atmospheric turbulence and the leading edge of the blade, the function $\Pi$ corresponds to the two-dimensional energy spectrum, that is modeled using a von Kármán spectrum for homogeneous and isotropic turbulence [4]. For trailing edge noise, 
corresponding to the scattering of the turbulent boundary layer at the trailing edge, $\Pi(\omega)=\Phi_{p p}(\omega) l_{y}(\omega)$, with $\Phi_{p p}(\omega)$ the wall pressure fluctuation spectrum, estimated with the Rozenberg et al. 23] model, and $l_{y}(\omega)$ the transverse correlation length calculated by Corcos model.

The expression (1) is valid for an airfoil that is fixed with respect to the receiver. For a rotating blade at the angular position $\beta$, the PSD at a far-field receiver at angular frequency $\omega$ is written [24, 4] :

$$
S_{p p}^{R}\left(\mathbf{x}_{\mathbf{R}}^{\mathbf{T}}, \omega, \beta\right)=\frac{\omega_{e}}{\omega} S_{p p}^{F}\left(\mathbf{x}_{\mathbf{R}}^{\mathbf{B}}, \omega_{e}, \beta\right)
$$

with $\omega_{e}$ the emission angular frequency, $\mathbf{x}_{\mathbf{R}}^{\mathbf{T}}$ the receiver coordinates in the wind turbine reference system, and $\mathbf{x}_{\mathbf{R}}^{\mathbf{B}}$ the receiver coordinates in the blade reference system. The superscript $R$ in Equation (2) refers to the rotating airfoil. The expression for the Doppler factor $\omega / \omega_{e}$ is given in Sinayoko et al. 24, who also derived an expression for the azimuthally averaged spectrum:

$$
S_{p p}^{a v}\left(\mathbf{x}_{\mathbf{R}}^{\mathbf{T}}, \omega\right)=\frac{1}{2 \pi} \int_{0}^{2 \pi} \frac{\omega_{e}}{\omega} S_{p p}^{R}\left(\mathbf{x}_{\mathbf{R}}^{\mathbf{T}}, \omega, \beta\right) \mathrm{d} \beta=\frac{1}{2 \pi} \int_{0}^{2 \pi}\left(\frac{\omega_{e}}{\omega}\right)^{2} S_{p p}^{F}\left(\mathbf{x}_{\mathbf{R}}^{\mathbf{B}}, \omega_{e}, \beta\right) \mathrm{d} \beta .
$$

To take into account the fact that the incident flow is not uniform along a wind turbine blade, a strip theory is used, that consists in cutting each blade into $M$ segments of variable chord $c_{m}$ and span $L_{m}$, so as to respect the condition $L_{m} / c_{m} \geq 3, m=1 . . M$, for which Equation (1) is valid. The different segments are supposed to be uncorrelated. A summation of the contributions for all blade segments are finally performed at the receiver. Note that all the expressions presented in this section are valid in free field in a homogeneous medium at rest.

\subsection{Coupling of Amiet's source model with an analytical propagation model for} the ground effect

The propagation of a point source over a finite impedance ground in a homogeneous atmosphere at rest is considered in this Section; see Figure 11(a). Assuming an $\mathrm{e}^{-\mathrm{i} \omega t}$ dependence, the sound pressure relative to the free field is 
classically written as [7]:

$$
\Delta L=\mathrm{SPL}-\mathrm{SPL}_{F F}=10 \log _{10}\left|1+Q \frac{R_{1}}{R_{2}} \mathrm{e}^{\mathrm{i} k_{0}\left(R_{2}-R_{1}\right)}\right|^{2},
$$

with SPL the sound pressure level, $\mathrm{SPL}_{F F}$ the free-field sound pressure level, $Q$ the spherical wave reflection coefficient, $k_{0}=\omega / c_{0}$ the acoustic wave number, $c_{0}$ the sound speed, and $R_{1}$ and $R_{2}$ the source receiver and image-source receiver distances. The spherical wave reflection coefficient is a function of the ground impedance and can be calculated analytically (see e.g. Refs. [7, 25, 26]). Using Equations (2) and (4), the sound pressure level for a rotating blade at the angular position $\beta$ above a finite impedance ground can be calculated as:

$$
\begin{aligned}
\mathrm{SPL}^{R}\left(\mathbf{x}_{\mathbf{R}}^{\mathbf{T}}, \omega, \beta\right) & =\mathrm{SPL}_{F F}^{R}\left(\mathbf{x}_{\mathbf{R}}^{\mathbf{T}}, \omega, \beta\right)+\Delta L=10 \log _{10}\left(\frac{S_{p p}^{R}\left(\mathbf{x}_{\mathbf{R}}^{\mathbf{T}}, \omega, \beta\right)}{p_{\text {ref }}^{2}}\right)+\Delta L \\
& =10 \log _{10}\left(\frac{S_{p p}^{R}\left(\mathbf{x}_{\mathbf{R}}^{\mathbf{T}}, \omega, \beta\right)}{p_{\text {ref }}^{2}}\left|1+Q \frac{R_{1}}{R_{2}} \mathrm{e}^{\mathrm{i} k_{0}\left(R_{2}-R_{1}\right)}\right|^{2}\right)
\end{aligned}
$$

with $p_{\text {ref }}=20 \mu \mathrm{Pa}$ the reference pressure.

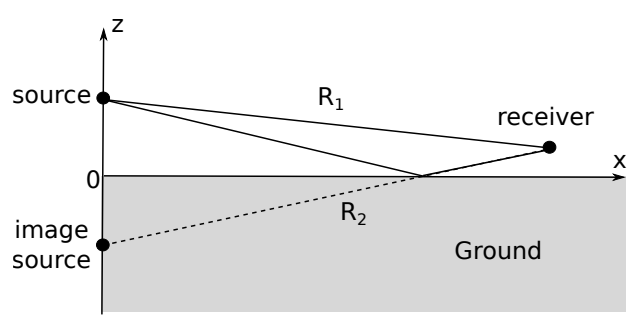

(a)

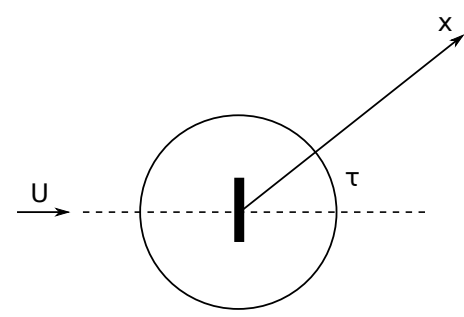

(b)

Figure 1: Notations for (a) the point source propagation over an infinite ground in a homogeneous atmosphere at rest, and (b) the wind turbine propagation in a direction $\tau$ with respect to the direction of the wind $U$.

\subsection{Amiet-PE coupled model}

Since the analytical model presented previously is only valid in a homogeneous atmosphere at rest, a propagation model based on the parabolic approximation is now presented to account for temperature and wind speed gradients in the atmosphere. The parabolic equation methods used are first presented 
in Section 2.3.1, and the coupling method with the aeroacoustic source model introduced in Section 2.1 is then detailed in Section 2.3.2.

\subsubsection{Acoustic propagation model based on the parabolic approximation}

There are different methods to obtain a wide-angle parabolic equation, in order to efficiently calculate the acoustic propagation in an inhomogeneous stratified atmosphere at rest [27, 28, 29, 7]. Two methods are compared in this study, namely a wide-angle parabolic equation based on a Padé $(1,1)$ approximation of the propagation operator and solved with the Crank-Nicholson method [27, 7], and a parabolic equation with fractional steps, called Split-Step Padé, based on higher order Padé approximants and solved with the method of Collins [29]. It may be noted that other parabolic equations exist for inhomogeneous moving atmospheres (see e.g. Refs [30, 31]). In this study, the effective sound speed approximation is used, which allows one to take into account the refraction effects due to the vertical wind gradients in the equation for a medium at rest [7]:

$$
c_{\mathrm{eff}}(z)=c(z)+U(z) \cos \tau=\sqrt{\gamma r T(z)}+U(z) \cos \tau
$$

with $\gamma$ the specific-heat ratio, $r$ the specific gas constant, $U(z)$ and $T(z)$ the mean vertical profiles of wind speed and temperature, and $\tau$ the angle between

the wind direction and the propagation direction noted as $x$, as shown in Figure $1(\mathrm{~b})$.

Using the axisymmetric approximation, the three-dimensional Helmholtz equation can be reduced to the following two-dimensional equation in the farfield [27, 7]:

$$
\left[\frac{\partial^{2}}{\partial x^{2}}+\left(\frac{\partial}{\partial z^{2}}+k^{2}\right)\right] q_{c}=0,
$$

where $q_{c}=p_{c} \sqrt{x}$ connects the $q_{c}$ variable to the complex pressure $p_{c}$, and $k$ is the acoustic wave number. This wave number can be written as $k^{2}=k_{0}^{2} n^{2}=$ $k_{0}^{2}(1+\epsilon)$, where $n(z)=c_{0} / c_{\mathrm{eff}}(z)$ is the index of refraction and $k_{0}$ is a reference value of the wave number. Introducing the propagation operator

$$
\mathcal{Q}=\left(1+\epsilon+\frac{1}{k_{0}^{2}} \frac{\partial}{\partial z^{2}}\right)^{1 / 2}=(1+\mathcal{L})^{1 / 2}
$$


we can decouple this equation into two equations characterizing a wave propagating in the positive $x$ direction, denoted as $q_{+}$(propagating wave), and a wave propagating in the negative $x$ direction denoted as $q_{-}$(backpropagating wave). Using the notation $\gamma= \pm 1$, one obtains from Equations (7) and (8):

$$
\left(\frac{\partial}{\partial x}-\mathrm{i} \gamma \mathcal{Q}\right) q_{\gamma}=0
$$

Introducing the variable $\phi_{\gamma}$ corresponding to the envelope of the pressure:

$$
q_{\gamma}(x, z)=\phi_{\gamma}(x, z) \exp \left(\mathrm{i} \gamma k_{0} x\right)
$$

and substituting Equation (10) into Equation (9), one gets:

$$
\frac{\partial \phi_{\gamma}}{\partial x}=\mathrm{i} \gamma(\mathcal{Q}-1) \phi
$$

The wide-angle parabolic equation (WAPE) is obtained by using a Padé $(1,1)$ approximant of the operator $\mathcal{Q}$ :

$$
\left(1+\frac{1}{4} \mathcal{L}\right) \frac{\partial \phi_{\gamma}}{\partial x}=\frac{1}{2} \mathrm{i} \gamma k_{0} \mathcal{L} \phi_{\gamma} .
$$

This expression is valid up to an elevation angle of $30-40^{\circ}$ [32, 7]. It can be solved by finite differences with the Crank-Nicolson method [27, 31, 7, discretizing the domain using a rectangular mesh of size $\Delta x$ and $\Delta z$ along $x$ and $115 z$ respectively.

To increase the angular validity of the parabolic equation, it is possible to use the Split-Step Padé $(\mathrm{N}, \mathrm{N})$ method proposed by Collins [29], noted in the following as $\operatorname{SSP}(\mathrm{N}, \mathrm{N})$. For $\gamma=+1$, the advancement scheme between the field at $x$ and at $x+\Delta x$ can be cast in the form [29, 33]:

$$
\phi_{1, n}=\frac{1+\mu_{n} \mathcal{L}}{1+\nu_{n} \mathcal{L}} \phi_{1, n-1}, \quad n=1 . . \mathrm{N},
$$

with $\phi_{1,0}(x, z)=\phi_{1}(x, z)$ and $\phi_{1, \mathrm{~N}}(x, z)=\phi_{1}(x+\Delta x, z)$. The advancement scheme for the wave propagating along $-x$ (where $\gamma=-1$ ) is obtained directly by inverting the coefficients $\mu_{n}$ and $\nu_{n}$ in Equation (13). The angular validity increases with the order $\mathrm{N}$ of the development and depends on the mesh size ${ }_{120} \Delta x$ chosen 33 . In the following, an order $\mathrm{N}=2$ is considered. As will be seen 
in the following, this method also makes it possible to use larger mesh sizes $\Delta x$, thus reducing the computation time.

Along the vertical direction, the domain is bounded by a ground impedance condition at $z=0$, and by an absorbing layer at the top of the domain to obtain non-reflecting boundary conditions [31, 17.

\subsubsection{Coupling of Amiet's source model with the PE code}

As explained in Section 2.1, each wind turbine blade is cut into $M$ segments. For each segment $m$, each angular position $\beta$ of the blade and each frequency $\omega$, a parabolic equation calculation is performed for which an initial condition at $x=$ 0 is needed. For this purpose, analytical solutions, typically of Gaussian type, are commonly used to represent the radiation of a monopole [27, 17. In order to preserve the directivity of the sources, the proposed coupling method uses initial numerical solutions based on the backpropagation method [34, 32, 35]. The principle, illustrated in Figure 2(a), consists first in back-propagating a known pressure field at $x=x_{i s}$ to $x=0$, taking $\gamma=-1$ in the Equations of Section 2.3.1. Then, in a second step, the starter at $x=0$ is forward-propagated to the desired distance using the "classical" parabolic equation with $\gamma=+1$.

In the backpropagation method, the initial solution at $x=x_{i s}$ is obtained for heights $z_{i s, p}=p \Delta z, p=0 . . P$, from the expression (2) for the SPL of a rotating blade. The initial solution thus includes the source directivity as viewed by this vertical line of receivers in this specific direction. In the absence of ground, it is written:

$$
q_{c}\left(z_{i s, p}\right)=\sqrt{S_{p p}^{R}\left(\mathbf{x}_{\mathbf{R}}^{\mathbf{T}}, \omega, \beta\right)} \sqrt{x_{S}} \mathrm{e}^{\mathrm{i} k_{0} R_{1, p}},
$$

with $R_{1, p}=\sqrt{x_{i s}^{2}+\left(y_{S}-y_{i s}\right)^{2}+\left(z_{S}-z_{i s, p}\right)^{2}}=\sqrt{r_{i s}^{2}+\left(z_{S}-z_{i s, p}\right)^{2}}$ the distance between the segment at $\left(x_{S}=0, y_{S}, z_{S}\right)$ and the $p^{\text {th }}$ initial starter point. Note that an extra term $\mathrm{e}^{\mathrm{i} k_{0} R_{1, p}}$ has been added to account for the geometrical phase shift due to differences in distance between the segment and the $p^{t h}$ initial starter point. In the presence of ground, the initial solution becomes:

$$
q_{c}\left(z_{i s, p}\right)=\sqrt{S_{p p}^{R}\left(\mathbf{x}_{\mathbf{R}}^{\mathbf{T}}, \omega, \beta\right)} \sqrt{x_{S}} \mathrm{e}^{\mathrm{i} k_{0} R_{1, p}}\left(1+Q \frac{R_{1, p}}{R_{2, p}} \mathrm{e}^{\mathrm{i} k_{0}\left(R_{2, p}-R_{1, p}\right)}\right),
$$




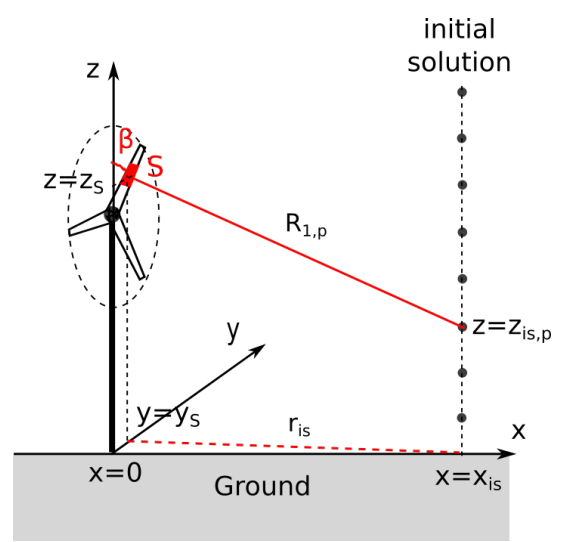

(a)

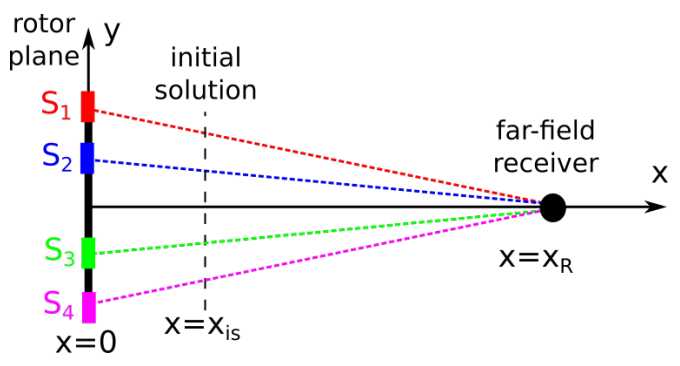

(b)

Figure 2: Schematics for (a) the backpropagation method used to obtain the starter at $x=0$ from the initial solution at $x=x_{i s}$ for one blade segment $S$, and (b) the different propagation planes between 4 blade segments noted $S_{1}, S_{2}, S_{3}$ and $S_{4}$ and the far-field receiver at $x=x_{R}$ (top view). Color online.

with $R_{2, p}=\sqrt{r_{i s}^{2}+\left(z_{S}+z_{i s, p}\right)^{2}}$ the distance between the image segment at $\left(x_{S}=0, y_{S},-z_{S}\right)$ and the $p^{t h}$ initial starter point .

During the backpropagation phase, the atmosphere is supposed homogeneous so that the analytical propagation solution can be used, and the ground is taken as rigid $(Q=1)$ because numerical instabilities may appear when backpropagating a field with an impedance ground [35. Then, the starter at $x=0$ can be propagated using any ground impedance and any sound speed profile. Note also that each calculation is performed in a slightly different plane that crosses the far-field receiver at $x=x_{R}$, as shown in Figure2(b). This method is therefore strictly exact only at this distance. For $x \neq x_{R}$, the SPL is obtained by summing contributions with different $y$ values. Since the radius of the rotor (typically $50 \mathrm{~m}$ ) is generally small compared to the propagation distances considered, the method will be seen to remain valid over a wide range of distances in Section 3 . 


\subsection{Point source approximation and effect of atmospheric absorption}

When the point source approximation is used, the wind turbine is represented as an omnidirectional point source of sound power level SWL $(\omega)$ located at the tower height $H$. This sound power level can be obtained from the sound pressure level in free field $\operatorname{SPL}_{F F}(\omega)$ in the direction $\tau$ using the azimuthally averaged spectrum of Equation (3):

$$
\begin{aligned}
\operatorname{SWL}(\omega) & =\operatorname{SPL}_{F F}(\omega)+10 \log _{10}\left(4 \pi R_{1}^{2}\right) \\
& =10 \log _{10}\left(\frac{S_{p p}^{a v}\left(\mathbf{x}_{\mathbf{R}}^{\mathbf{T}}, \omega\right)}{p_{\text {ref }}^{2}}\right)+10 \log _{10}\left(4 \pi R_{1}^{2}\right),
\end{aligned}
$$

where $S_{p p}^{a v}$ is obtained by summation over the segments of the three blades. Then, the sound pressure level is calculated using [7]:

$$
\operatorname{SPL}(\omega)=\operatorname{SWL}(\omega)-10 \log _{10}\left(4 \pi R_{1}^{2}\right)+\Delta L(\omega)-\alpha(\omega) R_{1},
$$

where the relative sound pressure level $\Delta L$ can be calculated using any propagation model, and the term $-\alpha(\omega) R_{1}$ corresponds to the atmospheric absorption, with $\alpha(\omega)$ the absorption coefficient in $\mathrm{dB} / \mathrm{m}$. This approximate way of including absorption effect is valid when the direct and reflected paths are approximately equal [7, i.e. $R_{2} \approx R_{1}$ in Figure 1(a). In the Amiet-PE coupled model, the effect of atmospheric absorption is also included in this approximate manner, by substracting a factor $\alpha(f) R_{1}$ to the sound pressure level at the farfield receiver. This approximation is justified when the distance of propagation is large with respect to the rotor diameter.

It is also possible to calculate the variation of SPL with the position of the blade $\beta$ during one rotation in the point source approximation. First, the "instantaneous" SWL is obtained from Equation (2):

$$
\begin{aligned}
\operatorname{SWL}(\omega, \beta) & =\operatorname{SPL}_{F F}(\omega, \beta)+10 \log _{10}\left(4 \pi R_{1}^{2}\right) \\
& =10 \log _{10}\left(\frac{S_{p p}^{R}\left(\mathbf{x}_{\mathbf{R}}^{\mathbf{T}}, \omega, \beta\right)}{p_{\text {ref }}^{2}}\right)+10 \log _{10}\left(4 \pi R_{1}^{2}\right),
\end{aligned}
$$

where $S_{p p}^{R}$ is obtained by summation over the segments of the three blades. 
Then, the "instantaneous" SPL is calculated as in Equation (17):

$$
\operatorname{SPL}(\omega, \beta)=\operatorname{SWL}(\omega, \beta)-10 \log _{10}\left(4 \pi R_{1}^{2}\right)+\Delta L(\omega)-\alpha(\omega) R_{1}
$$

This expression will be used to calculate the amplitude modulation of wind turbine noise in the point source approximation in Section 4.3 . Note that in Equation (19) the relative sound pressure level $\Delta L$ is calculated for a point source located at the tower height and is the same for all values of the angle $\beta$.

\section{Validation of the coupled model}

\subsection{Configuration studied}

In this study, the same 2.3 MW wind turbine as in Ref. [4 is considered, with a diameter of $93 \mathrm{~m}$, a mast height of $80 \mathrm{~m}$ and three blades of length $45 \mathrm{~m}$. As justified in Ref. [4, each blade is decomposed into 8 segments to respect the constraints mentioned in Section 2. The rotation of the blade is divided into 30 angular positions (resolution of $12^{\circ}$ ).

The wind velocity at $z=80 \mathrm{~m}$ is assumed to be $8 \mathrm{~m} / \mathrm{s}$, and the angular velocity of the rotor is $13 \mathrm{rpm}$. In this section, the following conditions are considered for validation purposes:

- source modeling : only trailing edge noise is included, and the wind speed profile is assumed to be constant (no shear effects) ;

- propagation modeling : the conditions are assumed to be homogeneous $\left(c(z)=c_{0}\right)$, with a finite impedance ground.

The absence of refraction effects makes it possible to compare the results of the coupled model with the analytical solution in a homogeneous atmosphere presented in Section 2.2 .

The ground impedance is calculated with a two-parameter variable porosity model, which is physically admissible and yields a better agreement with measurements than commonly used one-parameter models (e.g. Delany-Bazley or Miki), as shown by Dragna et al. [26]. The effective resistivity is $\sigma_{e}=50 \mathrm{kNs} / \mathrm{m}^{4}$ 
and the rate of change of the porosity is $\alpha_{e}=100 \mathrm{~m}^{-1}$. As shown in Ref. [26] based on the impedance measurements of [36, these are typical values for a natural soil. All the calculations are performed with this impedance ground, except in Section 3.2 where a perfectly rigid ground is also tested.

In order to calculate the third octave band SPL at the center frequency $f_{c}$ from a set of narrowband calculations, the following expression is used:

$$
\mathrm{SPL}_{1 / 3}\left(f_{c}\right)=10 \log _{10}\left[\frac{(\Delta f)_{1 / 3}}{N_{f}} \sum_{n=1}^{N_{f}} 10^{\operatorname{SPL}\left(f_{n}\right) / 10}\right],
$$

with $(\Delta f)_{1 / 3}=\left(2^{1 / 6}-2^{-1 / 6}\right) f_{c}$ the width of the third octave band centered at $f_{c}$. The number of narrowband calculations per third octave band are given in Table 1 for center frequencies between $100 \mathrm{~Hz}$ and $2000 \mathrm{~Hz}$. More calculations per third octave band are performed when the frequency increases, as classically done in the literature [37.

Table 1: Number of narrowband frequencies $N_{f}$ per third octave band for a center frequency $f_{c}$ between $100 \mathrm{~Hz}$ and $2000 \mathrm{~Hz}$.

\begin{tabular}{cccccccc}
\hline$f_{c}(\mathrm{~Hz})$ & 100 & 125 & 160 & 200 & 250 & 315 & 400 \\
$N_{f}$ & 1 & 1 & 1 & 2 & 2 & 3 & 4 \\
\hline$f_{c}(\mathrm{~Hz})$ & 500 & 630 & 800 & 1000 & 1250 & 1600 & 2000 \\
$N_{f}$ & 4 & 4 & 5 & 5 & 5 & 6 & 6 \\
\hline
\end{tabular}

Given the relatively high computation time of the coupled model, a simplified configuration denoted 1 and a complete configuration denoted 2 are considered ; see Table 2, In configuration 1, the domain is smaller and the spectrum is calculated on the third octave bands $100 \mathrm{~Hz}$ to $500 \mathrm{~Hz}$ only (i.e. $N_{f}=18$ narrowband calculations). The initial starter is computed at a distance $x_{i s}=$ $100 \mathrm{~m}$, greater than 30 acoustic wavelengths $\lambda$ over the whole spectrum.

\subsection{Validation with a single blade segment}

A series of calculations are made here with configuration 1, considering only the last segment of the 3 blades. Both rigid and impedance grounds are considered, and the effect of atmospheric absorption is neglected. The mesh size 
Table 2: Calculation parameters in configurations 1 and 2. $x_{R}$ is the position of the receiver and $N_{f}$ the number of calculated frequencies.

\begin{tabular}{lcc}
\hline & configuration 1 & configuration 2 \\
\hline$x_{R}$ & $500 \mathrm{~m}$ & $1000 \mathrm{~m}$ \\
domain size & $600 \mathrm{~m} \times 200 \mathrm{~m}$ & $1200 \mathrm{~m} \times 300 \mathrm{~m}$ \\
$N_{f}$ & 18 & 49 \\
third octave bands & $100 \mathrm{~Hz}$ to $500 \mathrm{~Hz}$ & $100 \mathrm{~Hz}$ to $2000 \mathrm{~Hz}$ \\
\hline
\end{tabular}

along $x$ is chosen equal to $\lambda / 10$ in the WAPE method, and it varies between $\lambda / 10$ and $5 \lambda$ in the $\operatorname{SSP}(2,2)$ method. The mesh size along $z$ remains equal to $\lambda / 10$ in both methods.
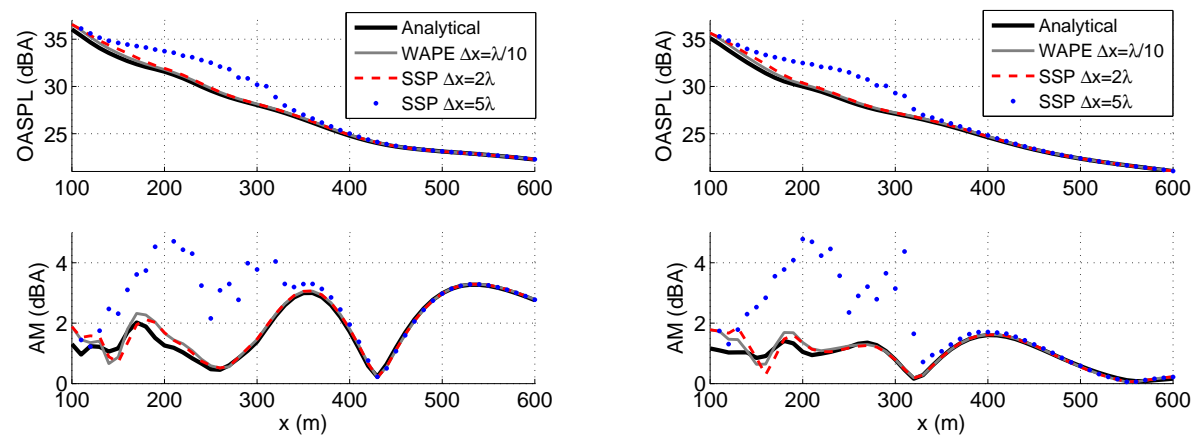

(a)

(b)

Figure 3: OASPL (top) and AM (bottom) as a function of $x$ at $z_{R}=2 \mathrm{~m}$ with $\tau=0^{\circ}$ and (a) a rigid ground or (b) an impedance ground for the analytical calculation (thick solid line), and for the coupled Amiet-PE calculations with the WAPE method and $\Delta x=\lambda / 10$ (gray solid line), or with the SSP $(2,2)$ method and $\Delta x=2 \lambda$ (dashed line) or $\Delta x=5 \lambda$ (dots). Color online.

Two important quantities to compare are the overall sound pressure level (OASPL) in $\mathrm{dB}(\mathrm{A})$, obtained by averaging over a rotation of the wind turbine, and the amplitude modulation (AM), corresponding to the difference between the maximum and the minimum of the OASPL over $\beta$. The evolution of these two quantities as a function of $x$ is plotted in Figure 3 for a receiver at a height of $2 \mathrm{~m}$ downwind $\left(\tau=0^{\circ}\right)$. The results are plotted only for $x \geq x_{i s}=100 \mathrm{~m}$. With 
the Amiet-PE and the analytical solution is obtained, especially between $300 \mathrm{~m}$ and $600 \mathrm{~m}$ where the curves superimpose perfectly.

Almost identical results are obtained with the $\operatorname{SSP}(2,2)$ method with mesh sizes between $\lambda / 10$ and $2 \lambda$; only the result with $2 \lambda$ is plotted in Figure 3 . With

below $350 \mathrm{~m}$. This is related to the reduced angular validity of the method with this time step, as can be seen in the plots of the OASPL and AM with respect to height (not shown here). These conclusions are valid for both rigid and impedance grounds. In the following, the calculations will be performed with the SSP $(2,2)$ method and a mesh size $\Delta x=2 \lambda$. The gain in computation time is about a factor 6 compared to the WAPE method with $\Delta x=\lambda / 10$.

The directivities of Figure 4 calculated in configuration 1 at a distance of $500 \mathrm{~m}$ from the wind turbine at a height of $2 \mathrm{~m}$ show that the OASPL and the $\mathrm{AM}$ are also well predicted in the other propagation directions, with a maximum observed in the near field [1, 4], the modulation amplitude is minimal downwind and upwind, where the OASPL is highest, and maximal close to the crosswind direction, where the OASPL is much lower. The higher AM obtained with a rigid ground can be attributed to the stronger interferences between direct and

\subsection{Validation for the entire wind turbine}

The calculations presented in this section considers all the blade segments in configuration 2, and includes the effect of atmospheric absorption, as described in Section 2.4. Due to the large number of frequencies $\left(N_{f}=49\right)$ and the greater

domain size compared to configuration 1, a configuration 2 computation takes approximately 4h30' to run for each segment and each direction $\tau$ on one core of a PC equipped with an Intel Xeon X5650 processor at 2.66 GHz. In order to reduce the computation time, it is possible to consider only the segments which are located towards the end of the blade, the segments close to the rotor having 


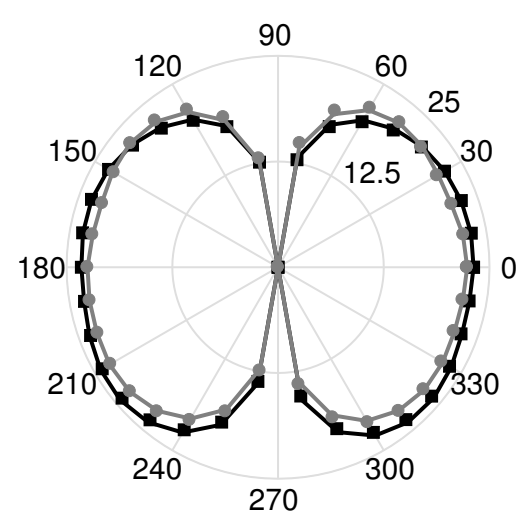

(a)

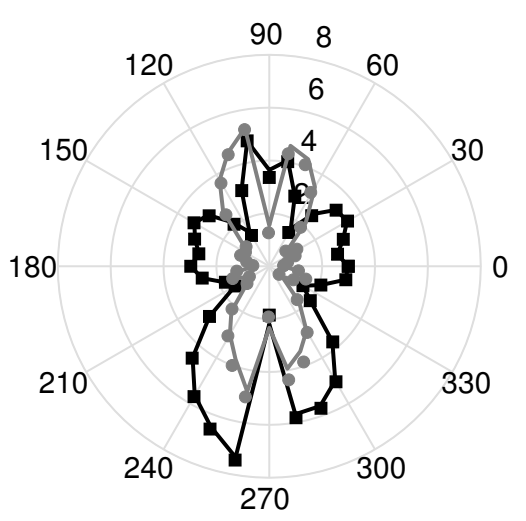

(b)

Figure 4: Horizontal directivity of (a) OASPL, and (b) AM for $x_{R}=500 \mathrm{~m}$ and $z_{R}=2 \mathrm{~m}$ for the analytical solution (symbols) and Amiet-PE calculations (lines) with a rigid ground (in black) and an impedance ground (in gray). The wind is coming from the left.

245 a low contribution to the total noise [38, 1. The third-octave band spectra of Figure 5 show that by considering only the segments 3 to 8 , the error is very small at $\tau=0^{\circ}$, and remains reasonable for most frequencies at $\tau=90^{\circ}$. The OASPL varies by less than $0.1 \mathrm{~dB}(\mathrm{~A})$ at $\tau=0^{\circ}$, and by less than $0.3 \mathrm{~dB}(\mathrm{~A})$ at $\tau=90^{\circ}$ when removing the first 2 segments. In the following, the calculations will be performed only with segments 3 to 8 .

The evolution of the OASPL and of the AM as a function of distance is plotted in Figure 6 for a downwind receiver $\left(\tau=0^{\circ}\right)$ and a crosswind receiver $\left(\tau=90^{\circ}\right)$. The OASPL predictions are in very good agreement with the analytical solution for all distances, i.e. even at large distances from the receiving point at $1000 \mathrm{~m}$ where all the planes shown in Figure 2(b) cross. The differences between AM analytical and numerical solutions are more visible, especially for $x<300 \mathrm{~m}$. As seen previously, the AM is relatively high in the crosswind direction, and remains smaller than $0.5 \mathrm{~dB}(\mathrm{~A})$ in the downwind direction. 


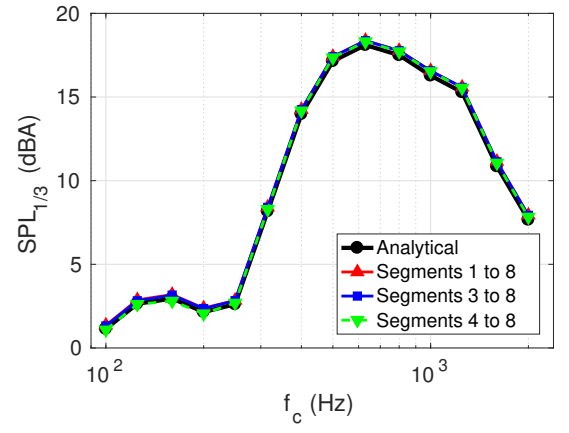

(a)

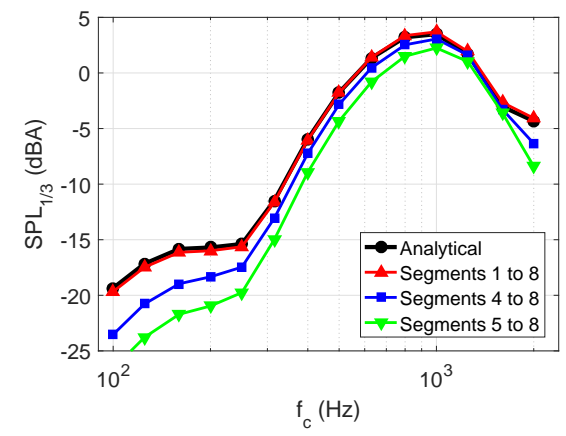

(b)

Figure 5: Third octave band spectrum of the sound pressure level at $x_{R}=1000 \mathrm{~m}$ and $z_{R}=2 \mathrm{~m}$ with (a) $\tau=0^{\circ}$ and (b) $\tau=90^{\circ}$ for the analytical and Amiet-PE calculations considering segments 1 to 8,3 to 8 and 4 to 8 . Color online.

\section{Results in an inhomogeneous atmosphere}

260

265 

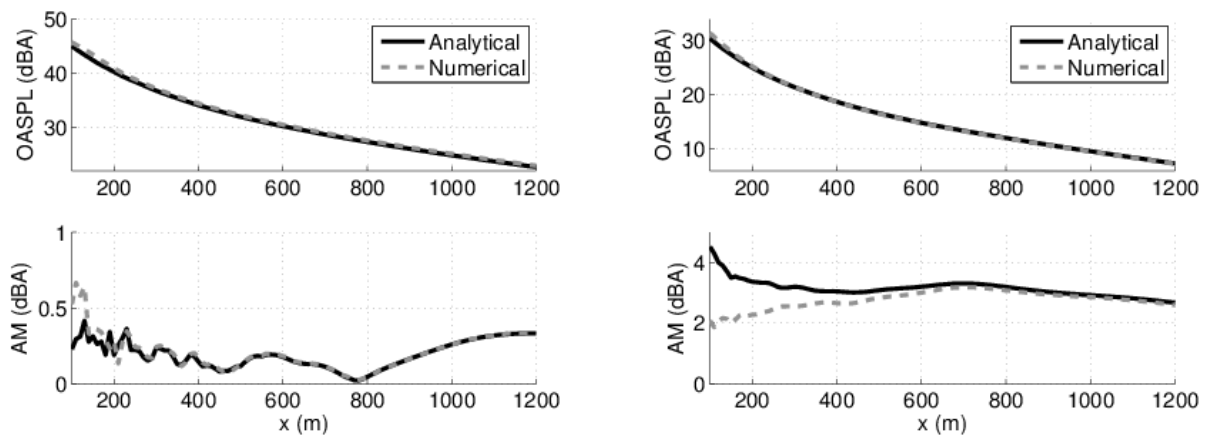

(a)

(b)

Figure 6: OASPL (top) and AM (bottom) as a function of $x$ at $z_{R}=2 \mathrm{~m}$ with (a) $\tau=0^{\circ}$ and (b) $\tau=90^{\circ}$ for the analytical calculation (solid line), and for the coupled Amiet-PE calculations with segments 3 to 8 (dashed line).

at $80 \mathrm{~m}$ height. The interested reader will find more information on the MoninObukhov similarity theory and on this choice of parameters in Reference [4] and in references cited therein.

Table 3: Parameters of Monin-Obukhov similarity theory for neutral $(H=0)$, stable $(H=$ $-25 \mathrm{~W} / \mathrm{m}^{2}$ and unstable $\left(H=200 \mathrm{~W} / \mathrm{m}^{2}\right)$ atmospheres.

\begin{tabular}{cccc}
\hline Atmosphere & $H\left(\mathrm{~W} / \mathrm{m}^{2}\right)$ & $u_{*}(\mathrm{~m} / \mathrm{s})$ & $L_{*}(\mathrm{~m})$ \\
\hline Neutral $(\mathrm{N})$ & 0 & 0.49 & Inf \\
Stable $(\mathrm{S})$ & -25 & 0.38 & 200 \\
Unstable $(\mathrm{U})$ & 200 & 0.58 & -92 \\
\hline
\end{tabular}
of effective sound speed for different propagation directions $\tau$ are plotted in Figure 7 for the three atmospheric conditions. It appears that the wind speed gradient between the bottom and the top of the rotor (between $35 \mathrm{~m}$ and $125 \mathrm{~m}$ approximately) is more important under stable conditions, typically occurring at night. Thermal gradients are strongest in the unstable atmosphere.

For these three atmospheric conditions, the third octave band spectra of sound power level can be calculated using Equation (16). The SWL depends on 

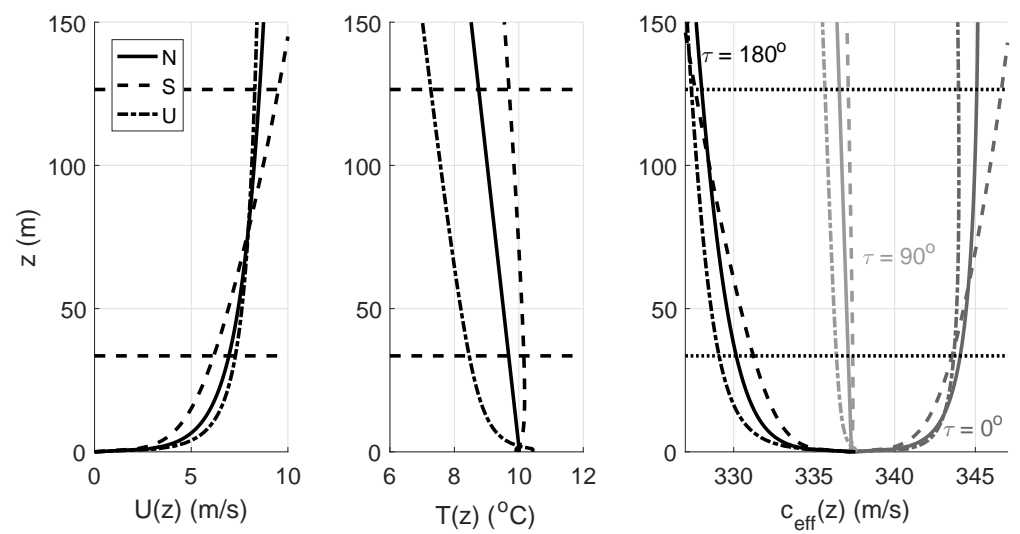

Figure 7: Vertical profiles of wind speed (left), temperature (middle), and effective sound speed for different directions $\tau$ (right) for a neutral atmosphere (solid lines), a stable atmosphere (dashed lines) and an unstable atmosphere (dashed-dotted lines). The minimum and maximum heights of the rotor are represented by horizontal dashed lines. Color online.

the direction of propagation considered, as shown in Figure 8 for $\tau=0^{\circ}$ and $\tau=$ $90^{\circ}$. The total SWL is much higher downwind than crosswind, and the spectral shape is also different. In both cases, turbulent inflow noise is the dominant noise mechanism at low frequencies (less than $300-400 \mathrm{~Hz}$ ) while trailing edge noise is dominant at higher frequencies. As was analyzed in Reference 4, the atmospheric conditions have a significant influence on turbulent inflow noise but they weakly modify the trailing edge noise spectrum. For this specific set of parameters, the SPL is higher in the unstable atmosphere compared to the stable and neutral atmospheres. However this result cannot be generalized because a weekly stable atmosphere has been considered here $\left(H=-25 \mathrm{~W} / \mathrm{m}^{2}\right)$. It has not been possible to consider more stable atmospheres (i.e. with $L_{*}<200 \mathrm{~m}$ ) since the Monin-Obukhov similarity theory is limited to very low heights in that case [4.

\subsection{Noise calculations for neutral, stable and unstable atmospheres}

The maps of the OASPL are plotted in $\mathrm{dB}(\mathrm{A})$ for a neutral atmosphere and for three directions of propagation in Figure $9(\mathrm{a}-\mathrm{c})$. It is clear that the levels 


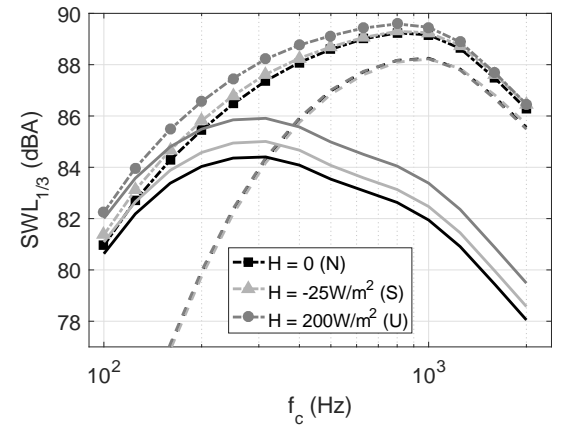

(a)

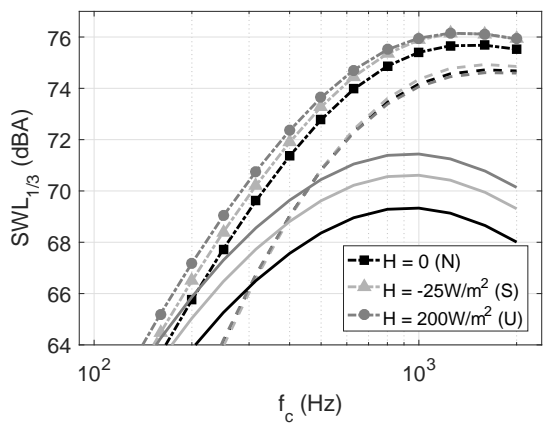

(b)

Figure 8: Third-octave band spectra of the sound power level in $\operatorname{dB}(\mathrm{A})$ at (a) $\tau=0^{\circ}$ and (b) $\tau=90^{\circ}$ for the three atmospheric conditions. The dashed lines correspond to trailing edge noise predictions, the solid lines to turbulent inflow noise predictions, and the dash-dotted lines with symbols to the total predictions. Color online.

crosswind $\left(\tau=90^{\circ}\right)$ are very low, due to the low SWL of the source in this direction. The levels downwind $\left(\tau=0^{\circ}\right)$ and upwind $\left(\tau=180^{\circ}\right)$ are similar up to about $500 \mathrm{~m}$. Beyond $500 \mathrm{~m}$, an acoustic shadow zone appears when the sound propagates upwind.

Figure 10 compares third-octave band spectra in different directions for a neutral atmosphere, at a distance of $500 \mathrm{~m}$ and $1000 \mathrm{~m}$. At $500 \mathrm{~m}$, one notices the modification of the ground effect due to atmospheric refraction. The interference dip is shifted to higher frequencies as $\tau$ increases, which explains the difference between downwind and upwind levels. At $1000 \mathrm{~m}$, the effect of the shadow zone is clearly felt for $\tau=135^{\circ}$ and $180^{\circ}$, with much lower levels than in the downwind direction, especially at high frequencies.

The OASPL is plotted as a function of the distance $x$ in Figure 11(a) for the three atmospheric conditions and for downwind $\left(\tau=0^{\circ}\right)$, crosswind $\left(\tau=90^{\circ}\right)$ and upwind $\left(\tau=180^{\circ}\right)$ receivers. The levels are higher in the upwind direction between $300 \mathrm{~m}$ and $600 \mathrm{~m}$ approximately, which can be attributed to the ground effect described above. Beyond $800 \mathrm{~m}$, on the other hand, the highest levels are observed downwind because upwind receivers are in the acoustic shadow zone. 


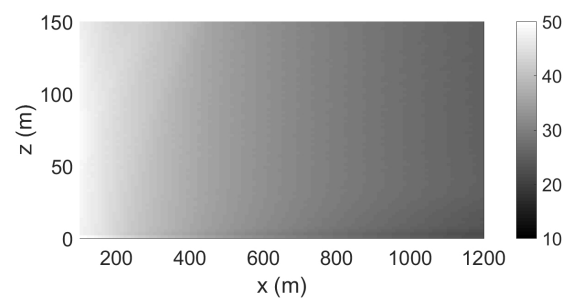

(a)

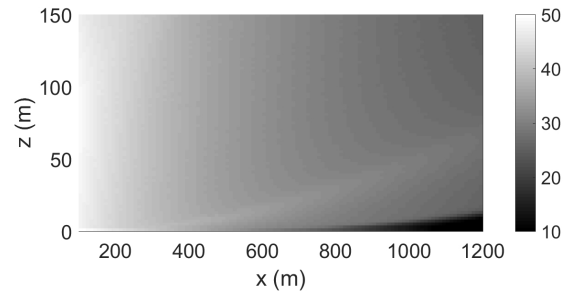

(c)

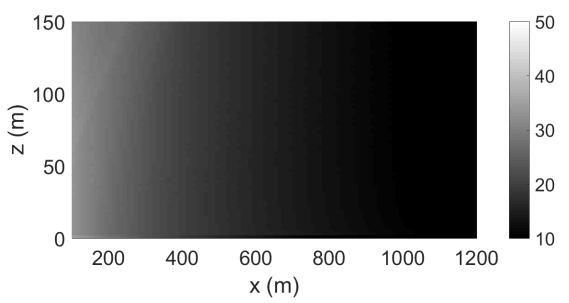

(b)

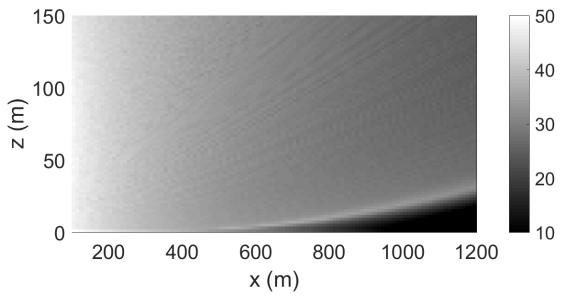

(d)

Figure 9: Maps of the OASPL in $\mathrm{dB}(\mathrm{A})$ for a neutral atmosphere $(H=0)$ with the Amiet-PE model at (a) $\tau=0^{\circ}$, (b) $\tau=90^{\circ}$, (c) $\tau=180^{\circ}$, and (d) with the point source approximation at $\tau=180^{\circ}$. Color online.

The difference between the levels in stable, unstable and neutral conditions is relatively low. The most significant difference is seen in the crosswind direction beyond $500 \mathrm{~m}$, where the level variations in the unstable atmosphere are different from the two other atmospheres due to the negative temperature gradients (see Figure 7).

The same type of plot is shown in Figure 11(b) for the amplitude modulation. The AM is very small downwind, and remains between 2 and $4 \mathrm{~dB}(\mathrm{~A})$ crosswind. For upwind receivers, the AM fluctuates in a complex way between 1 and almost $6 \mathrm{~dB}(\mathrm{~A})$ depending on distance and atmospheric conditions. To better understand these variations, it is interesting to look at the third octave band spectra of SPL and AM plotted in Figure 12 at $x_{R}=500 \mathrm{~m}$ and $1000 \mathrm{~m}$ upwind $\left(\tau=180^{\circ}\right)$. Because of diffraction effects, the shadow zone starts at shorter ranges at high frequencies. That is why for the receiver at $500 \mathrm{~m}$, only the highest frequencies are attenuated, while a large range of frequencies are attenuated for the receiver at $1000 \mathrm{~m}$. The largest AM occurs when the receiver 


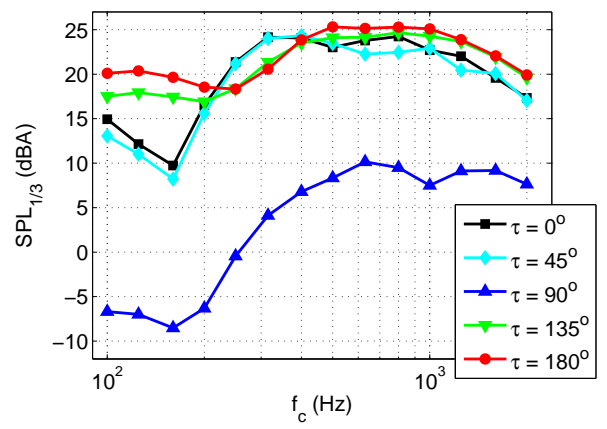

(a)

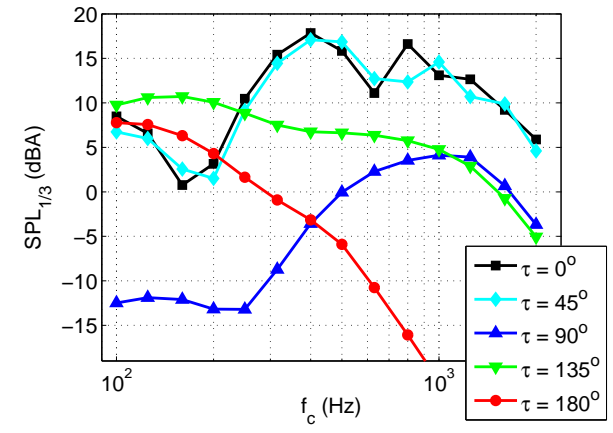

(b)

Figure 10: Third-octave band spectra of the sound pressure level in $\mathrm{dB}(\mathrm{A})$ at (a) $x_{R}=500 \mathrm{~m}$ and (b) $x_{R}=1000 \mathrm{~m}$ at a height of $2 \mathrm{~m}$ for a neutral atmosphere $(H=0)$. Color online.

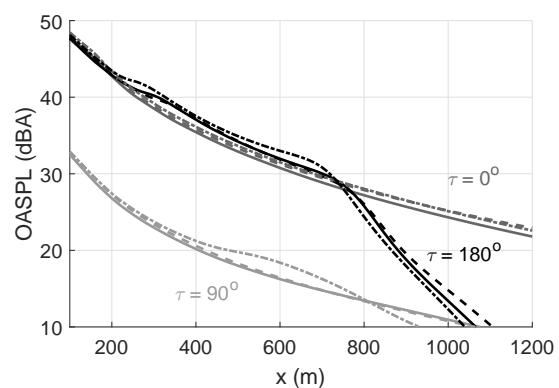

(a)

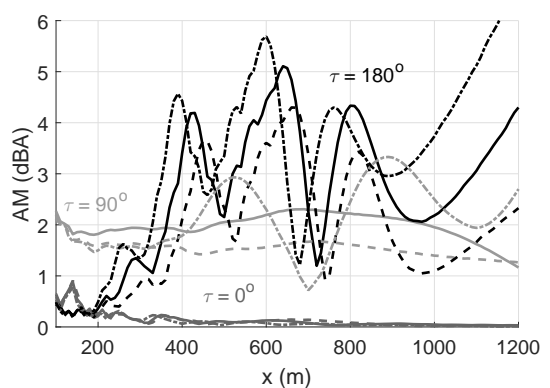

(b)

Figure 11: (a) OASPL and (b) $\mathrm{AM}$ in $\mathrm{dB}(\mathrm{A})$ as a function of $x$ at $z_{R}=2 \mathrm{~m}$ for a neutral atmosphere (solid lines), a stable atmosphere (dashed lines) and an unstable atmosphere (dash-dotted lines). Color online.

is close to the limit of the shadow zone at a given frequency, because it will enter and leave the illuminated region during the blade rotation. This explains why the highest $\mathrm{AM}$ are found at high frequencies at $500 \mathrm{~m}$, and at mid-frequencies at $1000 \mathrm{~m}$.

Figures 12(a) and (b) also show an interesting difference between the three atmospheric conditions in the upwind direction. Compared to the neutral and stable atmospheres, the SPL in the unstable atmosphere is $2 \mathrm{~dB}$ higher at low frequencies at a distance of $500 \mathrm{~m}$, and about $6 \mathrm{~dB}$ below over a large frequency 


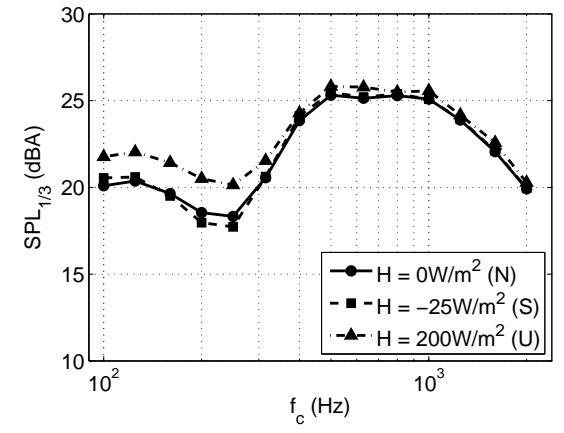

(a)

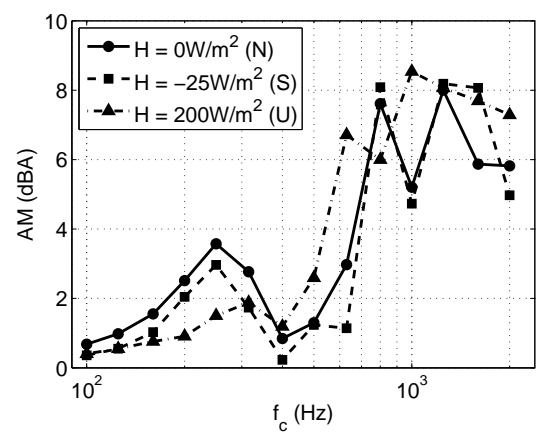

(c)

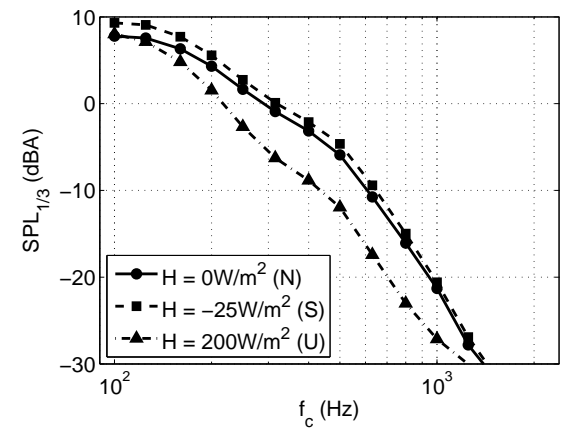

(b)

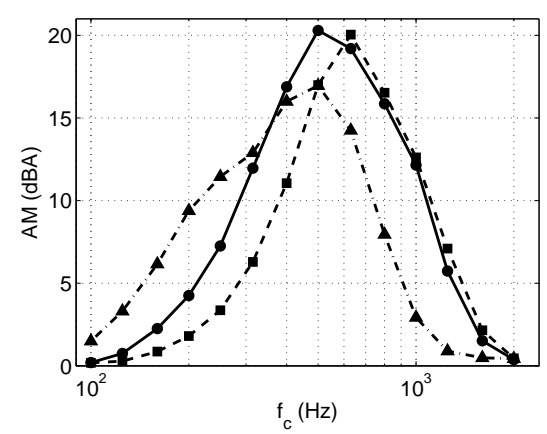

(d)

Figure 12: Third-octave band spectra of (a) the SPL at $x_{R}=500 \mathrm{~m}$, (b) the SPL at $x_{R}=$ $1000 \mathrm{~m}$, (c) the AM at $x_{R}=500 \mathrm{~m}$, and (d) the AM at $x_{R}=1000 \mathrm{~m}$, at a height of $2 \mathrm{~m}$ upwind $\left(\tau=180^{\circ}\right)$ for the three atmospheric conditions.

range at a distance of $1000 \mathrm{~m}$. This difference could be attributed to the strong sound speed gradient close to the ground in the unstable case, as shown in Figure 7 for $\tau=180^{\circ}$. The shadow zone starts at a shorter range in the unstable case, which explains the faster SPL decay at $1000 \mathrm{~m}$. At $500 \mathrm{~m}$, the ground effect is modified by the sound speed gradient, as already seen in Figure 10(a); this causes the interference dip to be shifted towards higher frequencies in the unstable atmosphere. 


\subsection{Point source approximation}

The calculations using the point source approximation are now compared to the Amiet-PE solution. Only the neutral atmosphere is considered. First, the third-octave band spectra are compared at a distance of $500 \mathrm{~m}$ and $1000 \mathrm{~m}$ for

Amiet-PE model, the shadow zone indeed starts at a higher range because of the various source heights considered in the calculation. The noise maps of Figure 9 (c-d) also show that the shadow zone is much larger when the point source approximation is used.

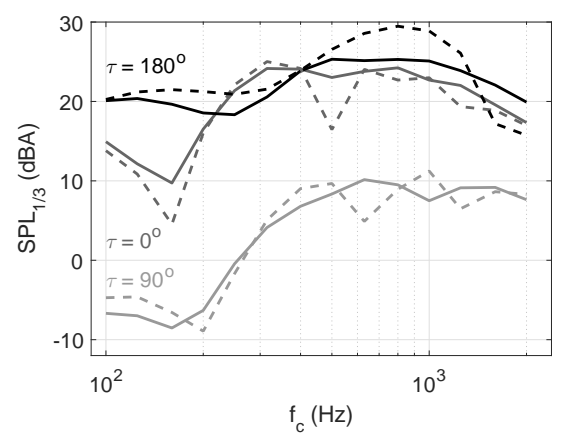

(a)

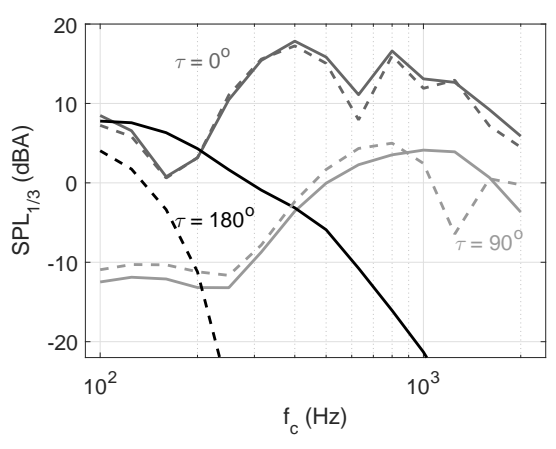

(b)

Figure 13: Third-octave band spectra of the sound pressure level in $\mathrm{dB}(\mathrm{A})$ for a neutral atmosphere at (a) $x_{R}=500 \mathrm{~m}$ and (b) $x_{R}=1000 \mathrm{~m}$ using the extended Amiet-PE model (solid lines) and the point source approximation (dashed lines). Color online.

The OASPL with the extended and point source models are compared in Figure 14(a) as a function of the distance $x$. Outside the acoustic shadow zone, the differences are relatively small. Due to the logarithmic summation, the strong interference dips appearing in the point source approximation result in a 


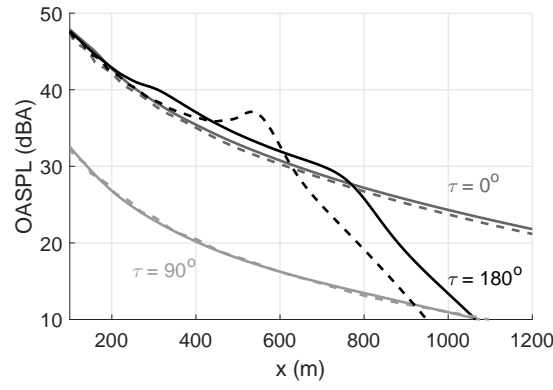

(a)

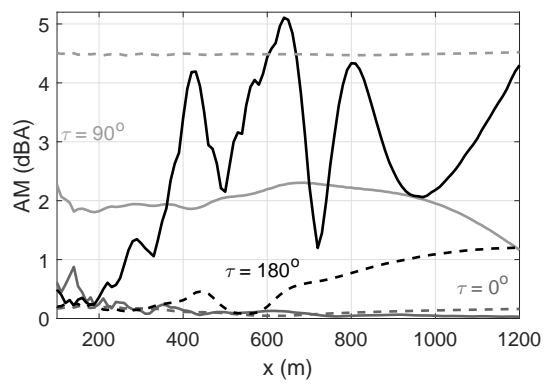

(b)

Figure 14: (a) OASPL and (b) AM in $\mathrm{dB}(\mathrm{A})$ as a function of $x$ at $z_{R}=2 \mathrm{~m}$ for a neutral atmosphere using the extended Amiet-PE model (solid lines) and the point source approximation (dashed lines). Color online.

small underestimation of the OASPL (up to $1.5 \mathrm{~dB}(\mathrm{~A})$ in the downwind direcincluded in the present model. This effect would tend to reduce the rate of decrease of the OASPL with distance in upward-refracting conditions [7, 37. It is most significant at high frequencies, since the shadow zone starts at shorter range for higher frequencies, as seen in the spectra of Figures 10 and 13 for

Neglecting atmospheric turbulence could also have an impact on amplitude modulation, although this effect has been much less studied in the literature. 
This topic would certainly deserve more attention in future studies, although performing noise propagation simulations with turbulence is very computationally demanding.

One classical method used to predict noise propagation in a refracting turbulent atmosphere is to synthesize various realizations of the turbulent fields and to study the statistics of the acoustic field through ensemble averaging [31, 7, 37. This method is limited to rather simple configurations (typically a homogeneous and isotropic turbulence over a flat ground) and requires at least 20 to 30 realizations to yield an accurate mean SPL. For more complex configurations, an alternative approach is to calculate the atmospheric flow with a time-domain Large Eddy Simulation (LES) and to perform PE simulations using LES velocity fields at different simulation times. This approach has been followed by Barlas et al. [17, 18 in order to study the effect of wind turbine wake velocity deficit and turbulence on sound propagation. The main difficulty is that LES calculates relatively large turbulence structures (e.g. spatial resolution of $2.5 \mathrm{~m}$ in Ref. [17]), thus omitting the scattering effect due to small-scale turbulence.

\section{Conclusions}

In this study, a method for coupling an aeroacoustic source model based on Amiet's theory and a parabolic equation code is proposed to model wind turbine noise propagation in an inhomogeneous atmosphere. The coupling method is based on the backpropagation method, that yields accurate initial conditions to the parabolic equation code while preserving the directivity of the aeroacoustic sources.

First, the proposed Amiet-PE model is validated by comparison with an analytical solution over a finite impedance ground in a homogeneous atmosphere, and a Split-Step Padé $(2,2)$ parabolic equation is shown to allow greater mesh sizes to be used and thus to reduce computation time. The third-octave band spectra, overall sound pressure levels and amplitude modulation are correctly predicted by the model. 
Then, wind turbine noise propagation is studied for different atmospheric conditions, considering both trailing edge noise and turbulent inflow noise sources. The Monin-Obukhov similarity theory is used to obtain the temperature and wind profiles, as well as the turbulence parameters needed as input to the turbulent inflow noise model. The main phenomenons observed are the modification of the ground effect due to atmospheric refraction, and the presence of a shadow zone when the receiver is against the wind at distances greater than about $800 \mathrm{~m}$. To understand the shape of the calculated third-octave band SPL spectra, it is necessary to account for the strength and directivity of the two aeroacoustic source mechanisms, and for the propagation effects related to ground reflection and atmospheric refraction.

Finally, the validity of the point source approximation has been assessed. The spectra obtained in this approximation tend to show interference dips that are averaged out in the coupled Amiet-PE model. The difference in the overall sound pressure levels predicted by the two models remains quite small, except for upwind receivers because the position of the shadow zone is not well predicted by the point source calculation. On the other hand, it is not possible to correctly predict the amplitude modulation using the point source approximation.

In the future, the Amiet-PE model could be used to understand the influence of more extreme atmospheric conditions, such as low level jets that are characterized by a wind speed profile with a maximum at a few hundred meters above the ground $[39,40$. Since the effect of atmospheric turbulence on noise propagation is neglected in the simulations presented in this article, it would be valuable to include this effect in the model, following for instance the turbulence synthesis method described in Ref. [37, in order to evaluate its effect on OASPL and AM. To be able to use the Amiet-PE model in impact studies, for instance to assess the efficiency of noise mitigation measures, it would be desirable to reduce its computation time. This could be done by considering 445 the blade segments as moving monopole sources, performing parabolic equation calculations only for a couple of source heights. This type of approach has been proposed recently by Barlas et al. [17] to study wind turbine wake effects on 
atmospheric sound propagation.

\section{Acknowledgments}

The author would like to thank Didier Dragna from École Centrale de Lyon for his advice on the backpropagation method and on the ground impedance model used in this article, and Benoit Gauvreau from IFSTTAR Nantes and Romain Monchaux from IMSIA for their useful comments on the manuscript.

\section{References}

[1] S. Oerlemans, J. G. Schepers, Prediction of wind turbine noise and validation against experiment, International Journal of Aeroacoustics 8 (2009) $555-584$.

[2] S. Oerlemans, M. Smith, P. White, S. von Hünerbein, A. King, B. Piper, M. Cand, A. Bullmore, B. Wilson, H. Madsen, A. Fischer, K. Kragh, Wind turbine amplitude modulation: Research to improve understanding as to its cause and effect, Technical Report, RenewableUK (2013) Available online: http://www.renewableuk.com/en/publications/reports.cfm/windturbine-amplitude-modulation.

[3] K. Heutschi, R. Pieren, M. Müller, M. Manyoky, U. W. Hayek, K. Eggenschwiler, Auralization of wind turbine noise: Propagation filtering and vegetation noise synthesis, Acta Acustica united with Acustica 100 (2014) 1324.

[4] Y. Tian, B. Cotté, Wind turbine noise modeling based on Amiet's theory: Effects of wind shear and atmospheric turbulence, Acta Acustica united with Acustica 102 (2016) 626-639. doi:10.3813/AAA.918979.

[5] F. Bertagnolio, H. Madsen, A. Fischer, A combined aeroelastic-aeroacoustic model for wind turbine noise: verification and analysis of field measurements, Wind Energy 20 (8) (2017) 1331-1348. doi:10.1002/we.2096 
[6] H. Hubbard, K. Shepherd, Aeroacoustics of large wind turbines, Journal of the Acoustical Society of America 89 (6) (1991) 2495-2508.

[7] E. M. Salomons, Computational Atmospheric Acoustics, Kluwer Academic Publishers, 2001.

[8] J. Prospathopoulos, S. Voutsinas, Application of a ray theory model to the prediction of noise emissions from isolated wind turbines and wind parks, Wind Energy 10 (2007) 103-119.

[9] K. Bolin, M. Almgren, E. Ohlsson, I. Karasalo, Long term estimations of low frequency noise levels over water from an off-shore wind farm, Journal of the Acoustical Society of America 135 (3) (2014) 1106-1114.

[10] S. Lee, D. Lee, S. Honhoff, Prediction of far-field wind turbine noise propagation with parabolic equation, Journal of the Acoustical Society of America 140 (2) (2016) 767-778.

[11] R. Makarewicz, Is a wind turbine a point source?, Journal of the Acoustical Society of America 129 (2) (2011) 579-581.

[12] G. van den Berg, Wind turbine power and sound in relation to atmospheric stability, Wind Energy 11 (2008) 151-169.

[13] F. Bertagnolio, H. Madsen, C. Bak, N. Troldborg, A. Fischer, Aerodynamic noise characterization of a full-scale wind turbine through high-frequency surface pressure measurements, International Journal of Aeroacoustics 14 (2015) 729-776.

[14] D. Ecotière, Can we really predict wind turbine noise with only one point source?, in: Sixth International Meeting on Wind Turbine Noise, Glasgow, United Kingdom, 2015.

[15] S. McBride, R. Burdisso, A comprehensive hamiltonian ray tracing technique for wind turbine noise propagation under arbitrary weather conditions, in: Seventh International Meeting on Wind Turbine Noise, Rotterdam, Netherlands, 2017. 
[16] F. Bertagnolio, H. Madsen, A. Fischer, Coupled wind turbine noise generation and propagation model: A numerical study, in: Seventh International Meeting on Wind Turbine Noise, Rotterdam, Netherlands, 2017.

[26] D. Dragna, K. Attenborough, P. Blanc-Benon, On the inadvisability of using single parameter impedance models for representing the acoustical 
properties of ground surfaces, Journal of the Acoustical Society of America 138 (4) (2015) 2399-2413.

${ }_{530}$ [27] M. West, K. Gilbert, R. Sack, A tutorial on the parabolic equation (PE) model used for long range sound propagation in the atmosphere, Applied Acoustics 37 (1992) 31-49.

[28] K. Gilbert, X. Di, A fast Green's function method for one-way sound propagation in the atmosphere, Journal of the Acoustical Society of America 94 (1993) 2343-2352.

[29] M. Collins, A split-step Padé solution for the parabolic equation method, Journal of the Acoustical Society of America 93 (4) (1993) 1736-1742.

[30] V. Ostashev, D. Juvé, P. Blanc-Benon, Derivation of a wide-angle parabolic equation for sound waves in inhomogeneous moving media, Acta Acustica united with Acustica 83 (1997) 455-460.

[31] L. Dallois, P. Blanc-Benon, D. Juvé, A wide-angle parabolic equation for acoustic waves in inhomogeneous moving media: Applications to atmospheric sound propagation, Journal of Computational Acoustics 9 (2) (2001) 477-494.

545 [32] M. Galindo, Approximations in the PE method. Phase and level errors in a downward refracting atmosphere, in: Seventh International Symposium on Long-Range Sound Propagation, Lyon, France, 1996.

[33] L. Dallois, P. Blanc-Benon, D. Juvé, The modelling of long range sound propagation: recent developments in the PE method, in: Tenth International Symposium on Long-Range Sound Propagation, 2002.

[34] M. Collins, E. Westwood, A higher-order energy-conserving parabolic equation for range-dependent ocean depth, sound speed, and density, Journal of the Acoustical Society of America 89 (3) (1991) 1068-1075. 
[35] D. Dragna, Modélisation par une approche temporelle de la propagation acoustique en milieu extérieur: traitement de frontières complexes et validation sur site ferroviaire [Time-domain modeling of outdoor sound propagation: treatment of complex boundary conditions and validation on a railway site], Ph.D. thesis, École Centrale de Lyon, no. 2011-35 (2011).

[36] G. Guillaume, O. Faure, B. Gauvreau, F. Junker, M. Bérengier, P. L'Hermite, Estimation of impedance model input parameters from in situ measurements: Principles and applications, Applied Acoustics 95 (2015) $27-36$.

[37] B. Cotté, P. Blanc-Benon, Estimates of the relevant turbulent scales for acoustic propagation in an upward refracting atmosphere, Acta Acustica united with Acustica 93 (6) (2007) 944-958.

[38] S. Oerlemans, P. Sijtsma, B. M. López, Location and quantification of noise sources on a wind turbine, Journal of Sound and Vibration (2007) 869-883.

[39] P. Baas, F. C. Bosveld, H. K. Baltink, A. A. Holtslag, A climatology of nocturnal low-level jets at Cabauw, Journal of Applied Meteorology and Climatology 48 (2009) 1627-1642.

[40] A. Lampert, B. B. Jimenez, G. Gross, D. Wulff, T. Kenull, One-year observations of the wind distribution and low-level jet occurrence at Braunschweig, North German Plain, Wind Energy 19 (2016) 1807-1817. doi: 10.1002/we.1951. 